The Encyclopedia of Chemistry

Edited by Prof. George L. Clark and Gessner G. Hawley. Pp. xvi+1037. (New York: Reinhold Publishing Corporation; London: Chapman and Hall, Ltd., 1957.) 156s. net.

7 HIS useful volume contains a very large amount of up-to-date material which it would be difficult to find elsewhere in such moderate compass. The articles are written by specialists and are, in general, very good. A wide field is covered and industrial applications receive careful treatment. The volume should be available in reference libraries. Since the book will no doubt go through many editions, it will not be out of place to refer briefly to what the reviewer thinks shortcomings. The index is most inadequate. The editors say that they kept the contributions strictly within the compass planned, and in some cases they have unduly cut down excellent articles and given far more space to poor ones than they deserved. In the accounts of persons and institutions the choice seems to be haphazard; although quite minor American institutions have long sections giving the names of present staff in full, the National Physical Laboratory and other famous foundations are not mentioned. Ostwald is mentioned but not Nernst, Herty but not Harden. Rutherford is still Sir Ernest and Aston is missing. In general, the editors do not seem to have been equal to their task. The spelling is sometimes weak; whether 'van der Waal', for example, is the responsibility of the author or is an editorial correction I do not know, but it should not appear.

J. R. Partington

\section{Elementary Practical Organic Chemistry}

By Dr. Arthur I. Vogel. Part 1: Small Scale Preparations. Pp. $x v+348+x i v$. (London and New York : Longmans, Green and Co., Ltd., 1957.) $21 s$.

П HE author has planned a three-volume work of which this is the first part. The book opens with forty pages of theory, followed by a long chapter on experimental technique. Besides dealing with customary purification processes, it contains an account of special small-scale apparatus - mostly modifications of familiar types used in macro-work-which the author has tried out and recommends. By standardizing the size of necks of flasks and the diameter of side-arms he has shortened the time required to set up an experiment. These devices and modifications have been carried out in co-operation with a large manufacturing firm, so that the standard apparatus may be obtained through laboratory suppliers. Many teachers will welcome the information in this chapter, for the text is punctuated with helpful notes on theory, comments on reagents and processes, laboratory devices and safety precautions. Chapters 3, 4 and 5 give practical directions for the preparation severally of a selection of aliphatic, aromatic and miscellaneous compounds. Although called 'elementary' these preparations are mostly of the class required by university students. They include one requiring the use of sodium borohydride and one reduction with lithium aluminium hydride. The publishers claim "that the requirements of all examinations involving a knowledge of practical organic preparations are fully met" and that the volume should be suitable for grammar schools, technical colleges and universities. However, it must be pointed out that "elementary organic prepara- tions", so far as British grammar schools are concerned, refer to such compounds as alcohol, acetone, acetic acid, chloroform, ether, ethylene, ethyl acetate and formic acid. The preparation of not one of these is given. Moreover, we are not told whether their omission implies that their preparation on a small scale is not expedient. Dr. A. I. Vogel believes that many of the preparations are new, and he states that all have been checked over in his laboratory. The advice on apparatus and technique, and the experience gone to the making of the book will commend it to teachers. Where it is not used as a class text it should be on the laboratory shelf.

\section{G. Fowles}

\section{Russian-English Glossary of Nuclear Physics and Engineering}

Pp. ii +195 . (New York : Consultants Bureau, Inc., 1957.) 10 dollars.

THIS excellent compilation gives idiomatic English equivalents of more than 10,000 Russian words and phrases. Its range goes beyond nuclear physics, nuclear engineering and atomic energy to include almost every technical word that might be encountered in Russian literature on those subjects. Errors are very few, and omissions are hard to find. There are some misprints in both Russian and English, but none is likely to cause serious difficulty. The main contribution to the list has been obtained by inverting various English-Russian dietionaries of similar scope, sometimes a little ineptly; it is evident in places that the Russian version is an explanatory translation of the English rather than an expression that might be used in scientific writing.

No grammatical information is included-not even an indication of parts of speech-and common words are not given, so that the glossary is of limited use to anyone not already familiar with Russian. The last page gives the Russian transliterations of various place names connected with work on atomic energy.

The binding is somewhat flimsy for a reference book. The glossary is described as the first of a series, from the same publishers, dealing with various branches of physics and ultimately to be included in "a comprehensive and authoritative dictionary of contemporary Russian terminology in all fields of modern physics". Such a work will be exceedingly valuable if the standard of this first instalment is typical.

J. B. SYKes

The United Kingdom Contribution to the International Geophysical Year, 1957-58

Pp. 72. (London: The Royal Society, 1957.) $10 s$.

7 HIS publication provides more than its title would lead one to expect. It contains a short, clear account of each subject to be studied during the International Geophysical Year, covering the observations to be made in each and the problems to be attacked. Each subject is linked with the others. Full details are given of the selection of the special periods, the observations to be made during them, and a calendar of those fixed in advance. Thus far the publication is international in scope, but finally we have also a record of the British share of the work with lists of those British scientists who have planned it. It is a book both to read and keep for reference on detrils. It is essential for all, professional scientists or laymen, who take a serious interest in the Geophysical Year.
G. A. BuLL 